\title{
NOTE
}

\section{Highly Conductive Polyfuran-13X Zeolite-Polyaniline Composite}

\author{
By Pinki SAHA SARDAR, ${ }^{1}$ Sanjib GHOSH, ${ }^{1}$ Mukul BISWAS, ${ }^{1}$ and Nirmalya BALLAV ${ }^{2, *}$
}

KEY WORDS: Composites / Polyfuran / Polyaniline / 13X-zeolite / Conductivity /

During the last few decades, polymer based hybrid organicinorganic composite materials have attracted a considerable research attention all over the world due to their extraordinary properties, which comes out of the synergism of distinctive properties of the components. ${ }^{1,2}$ While the incorporation of inorganic silicates (e.g., zeolites) as reinforcing fillers into various polymeric organic matrixes (e.g., elastomers) has been proved to be an efficient way of improving several properties of the resulting composite materials, ${ }^{1,3}$ the emergence of organic conducting polymers in the field has opened a novel and very important means to prepare advanced organic-inorganic functional materials. ${ }^{4,5}$ Among several $\pi$-conjugated conducting polymers (CPs), polyacetylene (PA), polyaniline (PANI), polypyrrole (PPY), and polythiophene (PTP) based composite materials have received a great deal of interest due to their high electrical conductivity and chemical and environmental stability. ${ }^{1,5-11}$ Significantly, CPs-based materials are being used these days as 'plastic electronics'11-14 (light emitting diodes (LEDs), photovoltaics, field-effect transistors (FETs)) and 'smart fluids'15,16 (usually called electrorheological fluids (ERFs) which are suspensions of polarizable particles dispersed in an insulating matrix).

However, owing to difficulty in synthesizing a regular $\pi$ conjugated structure with high conductivity, polyfuran (PF) has attracted relatively less attention. It is reported that PF exhibits some distinctive properties such as electrochromic effect and good redox ability. ${ }^{17}$ Moreover the resource of furan $(F)$ is much greater and thus $\mathrm{F}$ is much less expensive than its $\mathrm{N}$ and $\mathrm{S}$ analogues pyrrole (PY) and thiophene (TP) respectively. These characteristics should attract the attention of more scientists in chemistry and materials science. In the past decades, the electrochemical synthesis and properties of PF, co-PF-PPY, coPF-PTP biopolymer films have been studied. ${ }^{18-20}$ But it is observed that due to high oxidation potential of $\mathrm{F}$, a significant cleavage of the ring generally occurred during the electropolymerization, hence, strongly breaking the expected $\pi$ conjugated structure. The polymer obtained is dielectric or very low electrically conductive and the yield of the PF formed was greatly restricted by the area of the electrode used. Therefore, a chemical oxidative polymerization (COP) is needed. Recently Li et al. ${ }^{21}$ developed a combinatorial approach to synthesize PF and co-PF-PANI composite via COP with variable electrical properties. Ballav et al. ${ }^{22,23}$ reported preparation and characterization of $\mathrm{PF}-\mathrm{Al}_{2} \mathrm{O}_{3}, \mathrm{PF}-\mathrm{MMT}$, and PF-AB composite systems using COP. Interestingly $\mathrm{PF}-\mathrm{Al}_{2} \mathrm{O}_{3}, \mathrm{PF}-\mathrm{MMT}$ and $\mathrm{PF}-\mathrm{AB}$ composites showed improved thermal stability and conductivity characteristics respectively compared to bare PF. Several other interesting properties like hopping-transport ${ }^{24}$ and some theoretical calculations such as time-dependent density functional theory (DFT) calculations on band gaps and effective conjugation length of PF have also been studied ${ }^{25}$ during recent times. Overall there is a limited literature dealing with $\mathrm{COP}$ of $\mathrm{F}$ and PF based organic inorganic hybrid composite systems.

In view of the above background, we have developed a simple method exploring COP to synthesize PF-13X zeolite composite. We have further reinforced PF-13X composite by conductive PANI moieties. Details on the polymerization, composite formation and evaluations of some distinctive bulk properties will be highlighted in this article.

\section{EXPERIMENTAL}

\section{Materials}

Furan (Fluka, Switzerland), and aniline (Lancaster, UK) monomers were distilled (cold water $0{ }^{\circ} \mathrm{C}$ was circulated through the reflux condenser) under reduced pressure at $30^{\circ} \mathrm{C}$ and stored in a dark cool $\left(-20^{\circ} \mathrm{C}\right)$ place before use. White 13X-zeolite powder (Fluka, Switzerland) was heated at $100{ }^{\circ} \mathrm{C}$ for $2 \mathrm{~h}$ prior to use. Anhydrous $\mathrm{FeCl}_{3}$ (Merck, Germany) and ammonium peroxydisulfate (PDS, Merck, Germany) were used as such. All other reagents and solvents were of analytical grade (AR) and used after necessary purifications.

\section{Preparation of PF-13X Composite}

A weighed amount of $13 \mathrm{X}$-zeolite was taken in a $50 \mathrm{~mL}$ conical flask containing $25 \mathrm{~mL}$ of $\mathrm{CHCl}_{3}$ and sonicated for $15 \mathrm{~min}$. A known volume of $\mathrm{F}$ was injected to it under continued stirring. Thereafter, a particular amount of $\mathrm{FeCl}_{3}$ was added to this suspension at a time and the reaction mixture was kept under stirring for $1 \mathrm{~h}$ at room temperature. $20 \mathrm{~mL}$ of acetone was added to stop the polymerization reaction and to remove excess of $\mathrm{FeCl}_{3}$. The precipitated mass was filtered and

\footnotetext{
${ }^{1}$ Department of Chemistry, Presidency College, 86/1 College Street, Kolkata-700073, India

${ }^{2}$ Angewandte Physikalische Chemie, Universität Heidelberg, Im Neuenheimer Feld 253, D-69120 Heidelberg, Germany

*To whom correspondence should be addressed (Tel: +49-6221-54-4933, Fax: +49-6221-54-4933, E-mail: Nirmalya.Ballav@urz.uni-heidelberg.de).
} 
washed thoroughly by $\mathrm{MeOH}$ followed by acetone. Finally the dark grey coloured mass was dried at $50^{\circ} \mathrm{C}$ under vacuum for $1 \mathrm{~h}$ and subsequently characterized as the PF-13X composite.

\section{Preparation of PANI-PF-13X Composite}

Weighed amount of PF-13X composite prepared as above was taken in a $50 \mathrm{~mL}$ conical flask containing $25 \mathrm{~mL} 2(\mathrm{M}) \mathrm{HCl}$ solution. A known volume of ANI monomer was added to these systems and stirred for $15 \mathrm{~min}$. Thereafter, a known quantity of PDS was added to these mixtures and stirring continued for further $1 \mathrm{~h}$. Excess $\mathrm{MeOH}$ was added, filtered and the residues were repeatedly washed with acetone to remove oligomers of PANI. Finally the dark green coloured mass was dried under vacuum at $50^{\circ} \mathrm{C}$ for $1 \mathrm{~h}$ and characterized as PANIPF-13X composite.

\section{Characterization}

FT-IR spectra of PF-13X and PANI-PF-13X composites were taken on a JASCO 680 Plus model instrument using $\mathrm{KBr}$ pellets. Thermogravimetric analysis (TGA) and differential thermal analysis (DTA) were performed on Schimadzu DT 40 instrument. For SEM analyses, 13X-zeolite and composite samples were sprayed on mica foil and sputter coated with gold layers and, then a scanning electron microscope (Model JMS 5200) was used to take the SEM images. Direct current (dc) conductivity along with its dependence on temperature was measured on pressed pellets $(1.3 \mathrm{~cm}$ diameter and $0.1 \mathrm{~cm}$ width) with silver coating, using conventional four-probe technique. Current-Voltage (I-V) characteristics were also evaluated. The unit for such measurements was manufactured by the Scientific Equipments and Services, Roorke, India, which comprised of a low constant current source (Model LCS-02), a digital microvoltmeter (Model DMV-001) and a PID controlled oven (Model PID-200).

\section{RESULTS AND DISCUSSION}

\section{General Features of Polymerization and Composite For- mation}

Some typical data on the COP of F vis-à-vis composite formation of PF with 13X-zeolite, and on the PANI reinforcement of PF-13X composite are also given in Table I. Depending upon the experimental conditions, in general, 68-80\% loading of PF was observed for the PF-13X composite. Under similar conditions, such loading of $\mathrm{PF}$ was also observed for both $\mathrm{PF}-\mathrm{Al}_{2} \mathrm{O}_{3}$ and $\mathrm{PF}-\mathrm{AB}$ composite systems. COP of ANI in presence of $\mathrm{PF}-13 \mathrm{X}$ dispersion in aqueous medium at room temperature was conducted using a mole ratio of 1.5:1 between oxidant (PDS) and monomer (ANI). ${ }^{26}$ Depending upon the initial feed of ANI, the loading of PANI could be varied between $12-65 \%$. Such features of ANI polymerization vis-àvis PANI-PF-13X composite formation is consistent with the features observed during PANI-PF-MMT composite formation. $^{27}$
Table I. Some typical data on the polymerization and composite formation

\begin{tabular}{|c|c|c|c|c|c|}
\hline $\begin{array}{c}\text { Entry } \\
\text { No. }\end{array}$ & \multicolumn{2}{|c|}{ Reactants (g) } & \multicolumn{3}{|c|}{ Products } \\
\hline & Furan & Anhy. $\mathrm{FeCl}_{3}$ & $13 \times(g)$ & Composite $(\mathrm{g})$ & Loading of PF (\%) \\
\hline 1. & 1.0 & 1.0 & 1.0 & 1.40 & 70.3 \\
\hline 2. & 2.0 & 2.0 & 2.0 & 2.75 & 68.7 \\
\hline \multirow[t]{3}{*}{3.} & 1.0 & 2.0 & 1.0 & 1.60 & 80.0 \\
\hline & \multicolumn{2}{|c|}{ Reactants (g) } & \multicolumn{3}{|c|}{ Products } \\
\hline & ANI & PDS & $\begin{array}{c}\text { PF-13Xa } \\
\text { (g) }\end{array}$ & $\begin{array}{c}\text { Composite } \\
\text { (g) }\end{array}$ & Loading of PANI (\%) \\
\hline 4. & 0.25 & - & 0.5 & 0.53 & 12.0 \\
\hline 5. & 0.50 & - & 0.5 & 0.96 & 47.9 \\
\hline 6. & 0.75 & - & 0.5 & 1.43 & 65.1 \\
\hline
\end{tabular}

afrom entry No. 3

Table II. FT-IR absorption peaks $\left(\mathrm{cm}^{-1}\right)$ of various composite systems

\begin{tabular}{cc}
\hline Materials & Observed peaks $\left(\mathrm{cm}^{-1}\right)$ \\
\hline PF-13X & $629.2,798.9,1632.9,1753.94(\mathrm{PF})$ \\
PANI-PF-13X & $623.4,797.4,1652.7,1732.7(\mathrm{PF})$ \\
& $813.8,1240,1300.2,1479.1,1572.2(\mathrm{PANI})$ \\
\hline
\end{tabular}

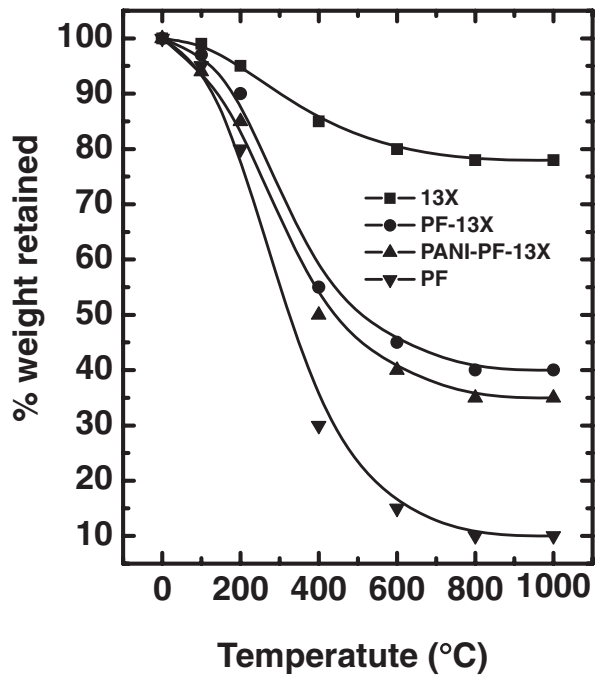

Figure 1. TGA scans for $13 X(\square)$, PF-13X(O) (loading of PF is $80 \%$ ), PANI$\mathrm{PF}-13 \mathrm{X}(\mathbf{\Delta})$ (loading of PF is $80 \%$ and PANI is $65 \%$ ) and $\mathrm{PF}(\boldsymbol{\nabla})$.

\section{FT-IR Spectral Analysis}

Table II presents FT-IR absorption peaks for PF-13X and PANI-PF-13X composite systems. The PF-13X composite showed FT-IR absorptions characteristic of PF. ${ }^{22,23}$ Further PF-13X composite, after reinforcement with PANI, showed characteristic FT-IR absorptions of both PF and PANI. ${ }^{27}$ Thus formation of these polymers and their subsequent incorporation in the respective $13 \mathrm{X}$ based composite systems was confirmed by FT-IR spectral analysis.

\section{Thermogravimetric Stability Studies (TGA/DTA)}

Figure 1 presents TGA scans for $13 \mathrm{X}(\boldsymbol{\square}), \mathrm{PF}-13 \mathrm{X}(\boldsymbol{O})$ (the loading of PF is $80 \%$; entry 3 of Table I), PANI-PF-13X( $\mathbf{\Delta})$ (loading of PF is $80 \%$ and PANI is $65 \%$; entry 6 of Table I) 

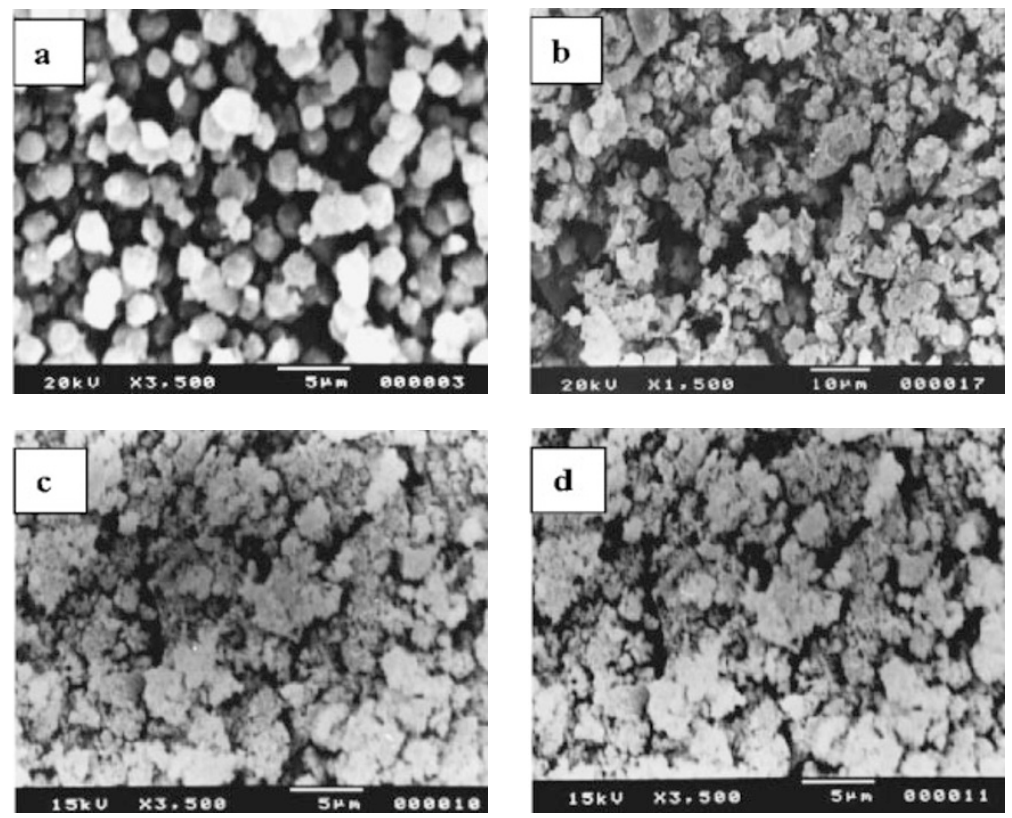

Figure 2. Scanning electron micrographs of $13 \mathrm{X}$ zeolite (a), PF-13X composite (b), and PANI-PF-13X composite ((c) \& (d)).

Table III. Thermal stability data (TGA/DTA)

\begin{tabular}{ccc}
\hline Materials & $\begin{array}{c}\text { \% Weight loss up to } \\
1000^{\circ} \mathrm{C}\end{array}$ & $\begin{array}{c}\text { Observed DTA peaks } \\
\left({ }^{\circ} \mathrm{C}\right)\end{array}$ \\
\hline $13 \mathrm{X}$ & 20 & - \\
PF & 90 & 255,351 \\
PF-13X & 60 & 355 \\
PANI-PF-13X & 65 & $397.8,450.2$ (shoulder) \\
\hline
\end{tabular}

a entry 3 of Table I, bentry 6 of Table I

and $\operatorname{PF}(\nabla)$ respectively and Table III summarizes the data on overall thermal stabilities (TGA and DTA) of these materials. In accordance with our earlier observations, PF showed almost complete loss $(90 \%)$ at around $1000{ }^{\circ} \mathrm{C}$ temperature and the remaining $10 \%$ could be due to a high temperature crosslinking reaction but the exact reason remains obscure. Interestingly, PF-13X showed an overall weight loss of only $60 \%$ at the similar temperature range. Such behavior of improve thermal stability is consistent with the results of PF- $\mathrm{Al}_{2} \mathrm{O}_{3}$ and PF-MMT composites. ${ }^{22,23}$ Regarding PANI-PF$13 \mathrm{X}$ composite, a further weight loss of $5 \%$ compared to PF$13 \mathrm{X}$ composite is observed which could be due to incorporation of thermally less stable PANI moieties in the comparatively more stable $\mathrm{PF}-13 \mathrm{X}$ composite. The overall thermal stability trend is as follows: $13 \mathrm{X}>\mathrm{PF}-13 \mathrm{X}>$ PANI-PF$13 \mathrm{X}>\mathrm{PF}$

The observed DTA peaks for PF-13X and PANI-PF-13X composites are due to exothermic oxidative degradation of polymeric moieties in the respective composites. Such peaks are characteristic of individual polymer backbones. A similar result was also obtained with PF-MMT and PANI-PF-MMT composites where DTA peaks characteristic for both PF and PANI were observed. ${ }^{27}$
Table IV. Dc conductivity values various composite systems

\begin{tabular}{ccc}
\hline Materials & Dc conductivity values $(\mathrm{S} / \mathrm{cm})$ & References \\
\hline PF-13X & $10^{-8}$ & This study \\
PANI-PF-13X & 1 & $"$ \\
PANI-PNVC-13X & $10^{-2}$ & 29 \\
PANI-PF-MMT & $10^{-3}$ & 27 \\
PANI-PMMA-SiO & $10^{-3}$ & 34 \\
PANI-PAN-SiO & $10^{-3}$ & 35 \\
PANI-PNVC- $_{2} \mathrm{O}_{3}$ & $10^{-3}$ & 36 \\
\hline
\end{tabular}

\section{Scanning Electron Microscopic Analysis (SEM)}

Figure 2 shows scanning electron microscopic (SEM) images of 13X (a), PF-13X composite (b) and PANI-PF-13X composite (c) and (d) respectively. In general, the SEM image of $13 \mathrm{X}$ zeolite showed nearly spherical particles with irregular sizes (average $2.0 \mu \mathrm{m}$ ). ${ }^{28,29}$ In contrast, SEM images of PF-13X composites indicated the formation of lumpy agglomerates of much larger particles (average $5.0 \mu \mathrm{m}$ ) with non-uniform morphology. Interestingly SEM images of PANI-PF-13X composite showed the presence of agglomerates of particles with more uniform morphological patterns than PF-13X composite. Formation of such aggregates could result through cementation of ultra-fine $13 \mathrm{X}$ particles with precipitating polymeric moieties (both PF and PANI) which is consistent with SEM images of various other polymer-metal oxide/ MMT/zeolite composite systems. ${ }^{2,30}$

\section{Conductivity and Current-voltage (I-V) Characteristics}

Table IV summarizes the dc conductivity values for PF-13X and PANI-PF-13X along with some other PANI reinforced composite systems. In general, $\mathrm{PF}-13 \mathrm{X}$ composite is essentially a non-conductive material like $\mathrm{PF}-\mathrm{Al}_{2} \mathrm{O}_{3}$ and PF-MMT composites. $^{22}$ However, a small loading of PANI (about 

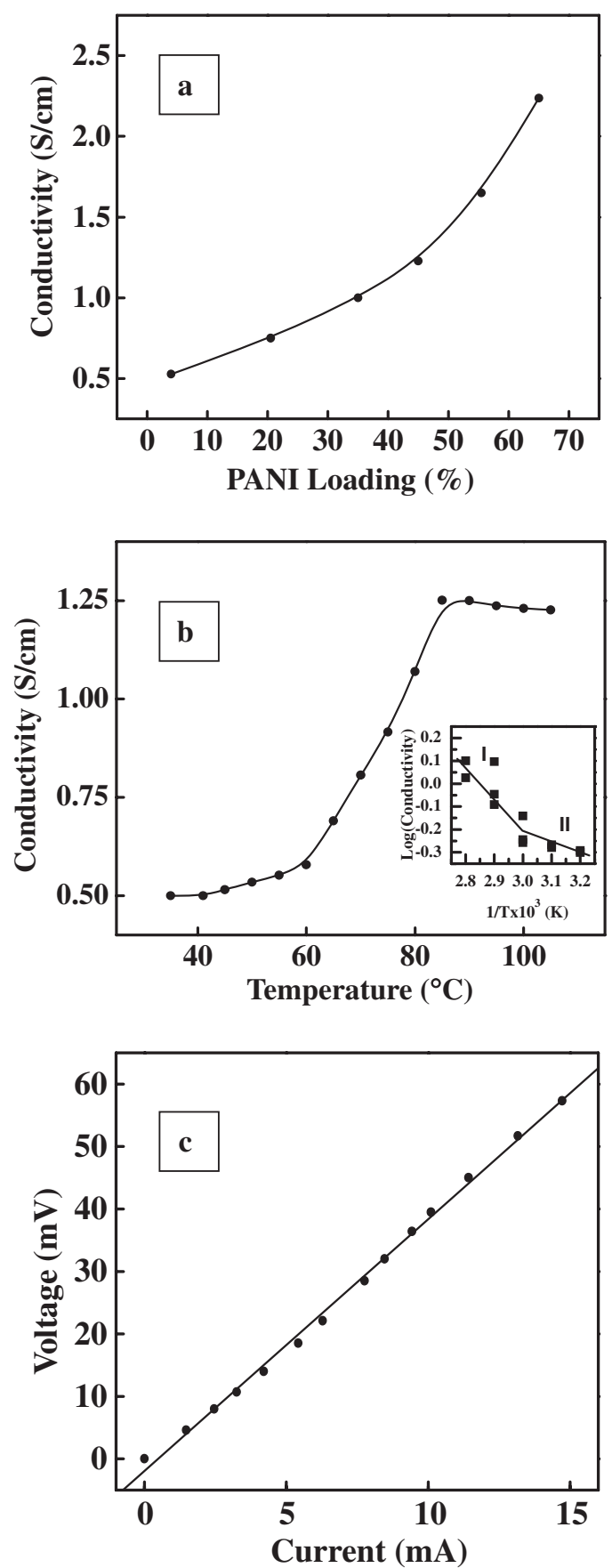

Figure 3. Conductivity characteristic of PANI-PF-13X composite as a function of PANI loading (a), and temperature (b). Current-voltage (I-V) curve for PANI-PF-13X composite (c).

$25 \%$ ) lead to a remarkable enhancement of the dc conductivity value for the resulted PANI-PF-13X composite (a $10^{8}$ fold increment). Interestingly, such improved conductivity was not obtained with other PANI reinforced composite systems. Probably this could be due to the following reasons. First, a good cementation of PANI moieties with PF-13X composites (see SEM images, compared to other PANI reinforced composite systems, a more uniform morphological pattern was obtained for the PANI-PF-13X composite. Secondly it could be due to 'entropy activation,' 31 i.e., polymerization of ANI took place on a more heterogeneous (less order, more entropy) PF-13X composite surface leading to a better distribution of PANI moieties (ordered, less entropy) over the whole system (more coverage).

Figure 3 represents dc conductivity values of PANI-PF-13X composite as a function of PANI loading (a) and temperature (b). Figure 3(a) reveals that with increasing PANI loading the conductivity value increases (non-linearity should be noted) which is expected. Figure 3(b) shows that with increasing temperature the conductivity value for the PANI-PF-13X composite increases (non-linear) and gets saturated (almost) beyond $90^{\circ} \mathrm{C}$. It is customary to give Log (conductivity) vs. $1 / \mathrm{T}$ plot (inset of Figure $3 \mathrm{~b}$ ) and we suggest activation energy of conduction of the composite in the order of $\sim 10^{-3} \mathrm{eV} / \mathrm{mol}$ as calculated from the slope of curve I (intrinsic conduction); curve II stands for impurity conduction (extrinsic conduction). Interestingly, the current-voltage (I-V) characteristic for the PANI-PF-13X (Figure 3(c)) shows a linear behavior which revealed the Ohomic nature of the composite. Thus, in view of potential applications, a dynamical correlation between reinforcement and temperature dependence, a desired I-V characteristic can be easily be adjusted for the PANI-PF-13X composite.

PF-13X composite was prepared and reinforced via incorporation of PANI moieties. The method involves in situ COP of ANI monomer in presence of an aqueous dispersion of preformed PF-13X composite. The dc conductivity of PF-13X composite could be improved significantly. This method can easily be extended for the reinforcement of several other polymer based organic-inorganic hybrid composite materials. Such 13X based binary composites are expected to behave as i) 'chemical sensors,' where synergetic interaction ${ }^{32}$ with various gases can be explored, ii) 'smart fluids, ' ${ }^{14}$ where dispersion of conductive polymers in an insulating matrix can be efficiently varied for better performance, and also as iii) 'antiferromagnetic' ${ }^{33}$ materials.

Acknowledgment. The authors thank the Department of Science \& Technology, Govt. of India, New Delhi, India, for funding a project (No. SR/S5/NM-14/2003) to MB and SG and a SRF position to PSS.

Received: August 3, 2008 Accepted: September 2, 2008 Published: October 16, 2008

\section{REFERENCES}

1. G. Kickelbick, Prog. Polym. Sci., 28, 83 (2003).

2. N. Ballav, Ph. D. Thesis, University of Calcutta, India, 2005.

3. A. M. S. Al-Ghamdi and J. E. Mark, Polym. Bull., 20, 537 (1988).

4. R. Gangopadhyay and A. De, Chem. Mater., 12, 608 (2000).

5. A. Maiti and M. Biswas, J. Ind. Eng. Chem., 12, 311 (2006).

6. V. Saxena and B. D. Malhotra, Curr. Appl. Phy., 3, 293 (2003).

7. N. Toshima and S. Hara, Prog. Polym. Sci., 20, 155 (1995).

8. J. Roncali, Chem. Rev., 92, 711 (1992).

9. A. G. Macdiarmid, Angew. Chem., Int. Ed., 40, 2581 (2001). 
10. A. G. Macdiarmid, Synth. Met., 125, 11 (2001).

11. P. K. H. Ho, J. S. Kim, J. H. Burroughes, H. Becker, S. F. Y. Li, T. M. Brown, F. Cacialli, and R. H. Friend, Nature, 404, 481 (2000).

12. M. Granström, K. Petrisch, A. C. Arias, A. Lux, M. R. Andersson, and R. H. Friend, Nature, 395, 257 (1998).

13. F. Würthner, Angew. Chem., Int. Ed., 40, 1037 (2001).

14. S. Sinha Ray and M. Okamoto, Prog. Polym. Sci., 28, 1539 (2003).

15. B. H. Kim, J. H. Jung, S. H. Hong, J. Joo, A. J. Epstein, K. Mizoguchi, J. W. Kim, and H. J. Choi, Macromolecules, 35, 1419 (2002).

16. J. H. Park, Y. T. Lim, and O. O. Park, Macromol. Rapid. Commun., 22, 616 (2001).

17. A. G. Shilabin and A. A. Entezami, Eur. Polym. J., 36, 2005 (2000).

18. G. M. Abou-Elenien, A. A. El-Maghraby, and G. M. El-Abdallah, Synth. Met., 146, 109 (2004).

19. X. B. Wan, W. Zhang, S. Jin, G. Xue, Q. D. You, and B. J. Che, J. Electro. Chem., 470, 23 (1999).

20. M. Kabaskaloglu, M. Talu, F. Yildirim, and B. Sari, Appl. Surf. Sci., 218, 84 (2003).

21. X. G. Li, Y. Kang, and M. R. Huang, J. Comb. Chem., 8, 670 (2006).

22. N. Ballav and M. Biswas, Polym. Int., 53, 1467 (2004).
23. N. Ballav and M. Biswas, Polym. Int., 54, 725 (2005).

24. G. R. Hutchsion, M. A. Ratner, and T. J. Marks, J. Am. Chem. Soc., 127, 2339 (2005).

25. J. Ma, S. Li, and Y. Jiang, Macromolecules, 35, 1109 (2002).

26. J. Stejskal and P. Kratochvil, Polymer, 37, 367 (1996).

27. N. Ballav, P. S. Sardar, S. Ghosh, and M. Biswas, J. Mater. Sci., 41, 2959 (2006).

28. N. Ballav and M. Biswas, Mater. Sci. Eng., B, 129, 270 (2006).

29. A. Maity, N. Ballav, and M. Biswas, J. Appl. Polym. Sci., 101, 913 (2006).

30. N. Ballav, A. Maity, and M. Biswas, Indian J. Eng. Mater. Sci., 10, 292 (2003).

31. S. Shu, S. Husain, and W. J. Koros, J. Phys. Chem. C 111, 652 (2007).

32. N. Densakulprasert, L. Wannatong, D. Chotpattananont, P. Hiamtup, A. Sirivat, and J. Schwank, Mater. Sci. Eng., B, 117, 276 (2005).

33. H. L. Frisch, H. Song, J. Ma, M. Rafailovich, S. Zhu, N. L. Yang, and X. Yan, J. Phys. Chem. B 105, 11901 (2001).

34. A. Maity and M. Biswas, J. Ind. Eng. Chem., 12, 626 (2006).

35. A. Maity and M. Biswas, Polym. Int., 54, 710 (2005).

36. A. Maity and M. Biswas, J. Appl. Polym. Sci., 94, 803 (2004). 